\title{
DYNAMIC AND STATIC STABILITY IN HEXAPEDAL RUNNERS
}

\author{
L. H. TING ${ }^{1}$, R. BLICKHAN ${ }^{2}$ AND R. J. FULL ${ }^{1, *}$ \\ ${ }^{1}$ Department of Integrative Biology, University of California at Berkeley, Berkeley, \\ CA 94720, USA and ${ }^{2}$ A.G. Nachtigall, Zoologie, Universität des Saarlandes, D-66041 \\ Saarbrücken, Germany
}

Accepted 2 August 1994

\begin{abstract}
Summary
Stability is fundamental to the performance of terrestrial locomotion. Running cockroaches met the criteria for static stability over a wide range of speeds, yet several locomotor variables changed in a way that revealed an increase in the importance of dynamic stability as speed increased. Duty factors (the fraction of time that a leg spends on the ground relative to the stride period) decreased to 0.5 and below with an increase in speed. The duration of double support (i.e. when both tripods, or all six legs, were on the ground) decreased significantly with an increase in speed. All legs had similar touchdown phases in the tripod, but the shortest leg, the front one, lifted off before the middle and the rear leg, so that only two legs of the tripod were in contact with the ground at the highest speeds. Per cent stability margin (the shortest distance from the center of gravity to the boundaries of support, normalized to the maximum possible stability margin) decreased with increasing speed from $60 \%$ at $10 \mathrm{~cm} \mathrm{~s}^{-1}$ to values less than zero at speeds faster than $50 \mathrm{~cm} \mathrm{~s}^{-1}$, indicating instances of static instability at fast speeds. The center of mass moved rearward or posteriorly with respect to the base of support as speed increased. Moments about the center of mass, as shown by the center of pressure (the equivalent of a single 'effective' leg), were variable, but were balanced by opposing moments over a stride. Thus, hexapods can exploit the advantages of both static and dynamic stability. Static or quasi-static assumptions alone were insufficient to explain straight-ahead, constant-speed locomotion and may hinder discovery of behaviors that are dynamic, where kinetic energy and momentum can act as a bridge from one step to the next.
\end{abstract}

\section{Introduction}

Stability during locomotion is a principal problem for most pedestrian animals. Unfortunately, our bias towards large, fast-moving mammals prevents us from appreciating the potential magnitude of the difficulty. The problem of instability is revealed by a simple calculation comparing a dog and a cockroach (Alexander, 1982). Assume an animal's body is a distance $h$ from the ground. If the body were suddenly

*To whom reprint requests should be addressed.

Key words: locomotion, mechanics, arthropods, cockroach, Blaberus discoidalis. 
unsupported, it would fall a distance $\boldsymbol{g} t^{2} / 2$ in time $t$, where $\boldsymbol{g}$ is the acceleration due to gravity. The body would fall a distance $h$ and hit the earth in time $(2 h / g)^{1 / 2}$. A dog walking slowly with its body $0.5 \mathrm{~m}$ above the ground at a stride frequency $(f)$ of $1 \mathrm{~Hz}$ would take one-third of a stride period [i.e. $f(2 h / g)^{1 / 2}$ ] before it hit the ground if left unsupported. A slow-walking cockroach $1 \mathrm{~cm}$ above the ground cycling its legs at $2 \mathrm{~Hz}$ would fall to the ground in only one-tenth of a stride period. This comparison suggests that, under these conditions, it is crucial that arthropods maintain static stability by having at least three legs on the ground at the same time, whereas large mammals can rely on dynamic adjustments to avoid falling (Alexander, 1971). The necessity for static stability in insects has been given as one of the most important reasons why insects have six legs and use two alternating tripods of support during locomotion (Hughes, 1952).

Numerous investigators (Alexander, 1982; Delcomyn, 1984, 1985; Forsythe, 1983; Fourtner, 1976; Graham, 1985; Hughes, 1952; Manton, 1977) have discussed the stepping patterns that insects require to maintain static stability during locomotion, yet few have attempted to quantify static stability as a function of gait or variation in body form (Cruse, 1976; Hughes, 1957). Studies on slow-moving stick insects have begun to address the stability problems of animals walking straight ahead and around curves (Jander, 1985).

Research on legged walking machines provides an approach to quantifying static stability (McGhee, 1976; Song, 1984; Sun, 1974). The minimum requirement to achieve static stability is a tripod of support, as in a stool. If an animal's center of mass falls outside this triangle of support formed by its three legs on the ground, it is statically unstable and will fall. In the quasi-static gait of a robot or animal, the center of mass moves with respect to the position of the legs on the ground, and the probability of falling increases as the center of mass approaches the edge of the triangle of support. This probability has been quantified for all $n$-legged walking vehicles by calculating the longitudinal stability margin, the shortest distance from the center of mass to the boundary of the support pattern in front of or behind the vehicle. The longitudinal stability margin $(S)$ of a wave gait (i.e. a regular, symmetrical gait) normalized for stride length is described by:

$$
S=\left(n_{\operatorname{legs}} / 4\right) \beta-3 / 4,
$$

where $\beta$ is the duty factor, the fraction of a stride period that a leg is in the support phase and $n_{\text {legs }}$ represents the number of legs (Song, 1984). When $S<0$, the animal is statically unstable. A six-legged arthropod would become less stable as the duty factor decreased to 0.5 and unstable if it fell below 0.5 . This model has the advantage of comparing animals with different numbers of legs and different stepping patterns. Although the $n$-legged vehicular walking model is obviously an oversimplification for the requirements of an insect to maintain stability, it can nevertheless provide a basis for more complete models. Direct measurements of static stability in different locomotor designs can be obtained by examining the center of mass and its relationship to the base of support.

In the present study on the death-head cockroach Blaberus discoidalis, we measured the static stability margin (the minimum distance of the center of mass from the edge of the support triangle, not only longitudinally but also with respect to any side of the 
triangle). We attempted to reconcile the apparent static stability suggested by the design of insects with evidence of dynamic behavior found in recent studies. Investigations on the mechanics of locomotion in cockroaches (Full and Koehl, 1993; Full and Tu 1990, 1991; Full et al. 1991) and crabs (Blickhan and Full, 1987; Blickhan et al. 1993) have provided evidence of movement not limited by static constraints, but more similar to dynamic locomotion described in birds and mammals. Bodies of two-, four-, six- and eight-legged runners accelerate and decelerate due to remarkably similar ground reaction force patterns. Despite wide variation in morphology, running in cockroaches, crabs, birds and mammals can be described by a single model consisting of a bouncing springmass system (Blickhan and Full, 1993; Farley et al. 1993; Full, 1993). We recognize the importance of static stability at low speeds in hexapods, but hypothesize that determination of duty factors, leg phase relationships, stability margin and moments about the center of mass will reveal the significance of dynamic stability in insect locomotion.

\section{Materials and methods}

\section{Animals}

For stepping patterns and stability margin measurements, Blaberus discoidalis (Serville) were obtained from a supply company (Carolina Biological Supply). Average mass was $3.0 \pm 0.9 \mathrm{~g}$ (S.D., $N=3$ ). Animals were individually housed in plastic containers. They were fed dog chow and water ad libitum.

\section{Stepping patterns and stability margin}

Animals ran across an acrylic track as we video-taped them from above to determine stepping patterns during constant, average-velocity running (the sums of the increases and decreases in speed were within $\pm 10 \%$ of the average speed). The track was $6 \mathrm{~cm}$ wide, constraining the animals to approximately straight-ahead running. Cockroaches ran freely from one end of the track into a darkened box. The temperature of the trackway was $24^{\circ} \mathrm{C}$. Trials in which the animals touched the sides of the track were discarded.

Animals were taped using high-speed video at 250 or 500 frames s $^{-1}$ (Kodak Ektapro SE) to determine the position of the tarsus of each leg and their respective ground contact times. The primary source of illumination was a horizontal plane of light that projected across the bottom of the track so that long shadows of the legs would appear when they were close to the ground. The light was low enough that the body of the cockroach was above the plane of light and did not cast a shadow. When the end of the shadow was coincident with the ground-contact point on the tarsus, the leg was determined to be on the ground. The positions of the head, the abdomen and the tarsus of each leg were digitized into a computer using a motion analysis system (Peak Performance Technologies, Inc.). In addition, tabulations of when each leg was on or off the ground were made for each frame.

The positions of the legs and body in each frame were used to calculate the per cent stability margin of the animal during running, as well as the phase lag and duty factor for each leg. The cockroach is known to employ an alternating tripod gait at all but the very 
slowest speeds. The front and hind legs of one side move in synchrony with the second leg of the opposite side. Thus, the animal is statically stable when its center of mass of lies within the triangle of support formed by the legs in each tripod. The stability margin is defined by the minimum distance of the center of mass from the edge of the support triangle (McGhee, 1976; Song, 1984; Fig. 1A). The per cent stability margin represents the stability margin normalized by the maximum possible stability margin when each calculation was made, allowing comparisons among animals. The position where $100 \%$ stability would be achieved is termed the ideal center of stability. The phase of the leg is the fraction of a stride at which a leg touched or lifted off from the ground after the hind leg had touched down (i.e. a value of 0 represents the time in the cycle of hind leg touchdown and a value of 1 indictaes the time when the hind leg touches down again for the next stride). These data reveal how tightly coupled leg motion is in a tripod of support. The duty factor is the fraction of a stride period that the leg is on the ground providing support. We also calculated the distance of the center of mass from the ideal center of stability in the longitudinal direction (i.e. the direction of motion).

\section{Moment of inertia}

We measured the moment of inertia of the cockroach for three principal axes of rotation, corresponding to pitch $(x)$, yaw $(z)$ and roll $(y)$ of the body (Fig. 1B). Intact, frozen $B$. discoidalis were used to estimate the moment of inertia $(I)$. The total leg mass, excluding the coxa (which moves very little and is close to the body) was less than $5 \%$ total body mass, so legs contributed very little to the moment of inertia. An insect pin was inserted through the body of the cockroach parallel to the axis of rotation to be measured. We measured the distance from the pin to the center of mass of the animal (approximately $50 \%$ of body length) and recorded the animal's mass. The pin with the cockroach was then suspended on knife blades. The animal was lightly tapped and allowed to oscillate as

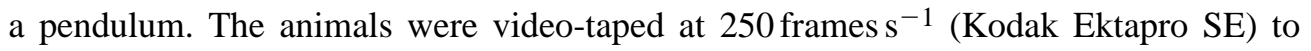
determine the period of oscillation. The moments of inertia about the $x$-, $y$ - and $z$-axes were calculated using the following formula:

$$
I_{\mathrm{axis}}=\frac{T^{2} m c \boldsymbol{g}}{4 \pi^{2}}-m c^{2},
$$

where $T$ represents the period of oscillation, $m$ is mass, $c$ is the distance from the center of mass to the pin insertion point and $\boldsymbol{g}$ is the acceleration due to gravity (Fedak et al. 1982).

\section{Moments about the center of mass and the center of pressure}

We combined single leg forces and kinematic data to estimate the center of pressure of the ground reaction forces on the body of the cockroach. The whole-body ground reaction forces are known to look similar to force patterns produced by other trotting animals (Full, 1991; Blickhan and Full, 1993). However, the line of action of the resultant force cannot be found from whole-body reaction forces because the individual leg forces were not resolved. With three legs on the ground, there is no unique set of leg forces that could have created the resultant. By combining single-leg force measurements with 


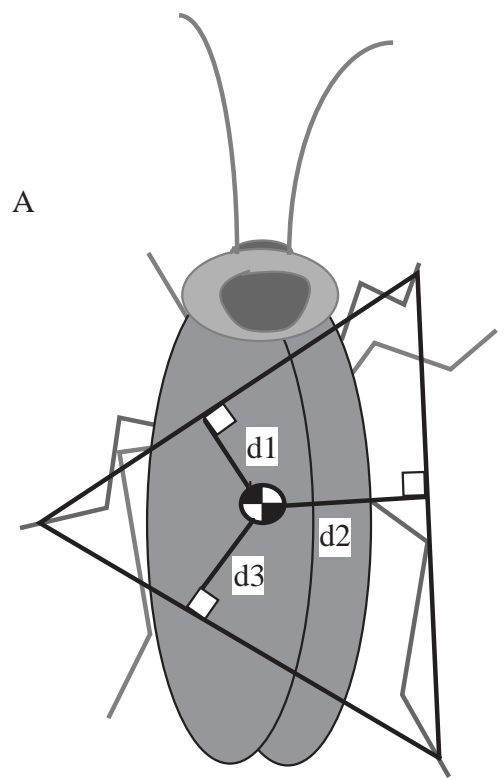

\section{Stability margin}

The shortest distance from the center of mass to the edge of the tripod of support.

\section{Ideal center of stability}

Position where the greatest

stability margin is attained

\section{Per cent stability margin}

Stability margin normalized to maximum

achievable stability margin

B
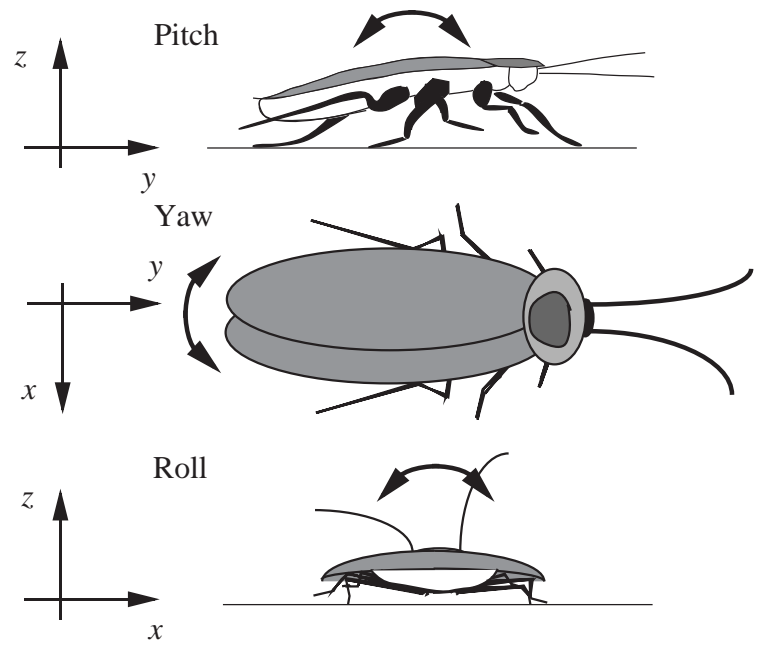

oll

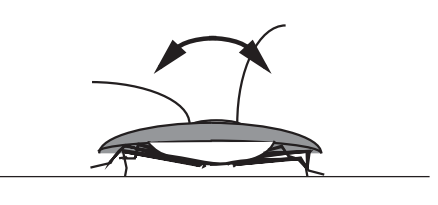

Fig. 1. (A) Static stability measures. Stability margin is equal to d1. The tripod of support is represented by the dark triangle. The black-and-white circle represents the center of mass. (B) Pitch $(x)$, yaw $(z)$ and roll $(y)$ axes.

measurements of the tibia-tarsus joint position, it was possible to calculate the center of pressure or the position where a single leg would act on the body during locomotion.

Measurements of single-leg ground reaction forces in B. discoidalis were taken from data collected by Full et al. (1991). Single-leg force data for the front, middle and rear legs obtained at similar speeds were used together in a single calculation of center of pressure. Forces from different trials were combined in several permutations to produce 
sets of force data for a tripod, which were then used with kinematic data of leg positions from separate, single trials to calculate moments about the center of mass. For each trial, two successive, symmetrical steps were considered, and the total moments generated about the center of mass of the body were calculated. The amount of double tripod support (i.e. the fraction of a stride during which six legs are on the ground, three from each tripod) was also varied in accordance with the kinematic data. The moment vector (M) is simply the cross-product of the distance (r) and force vectors $(\mathbf{F})$ associated with each tarsus (i.e. leg segment in contact with the ground) summed over all three legs on the ground at a given time:

$$
\mathbf{M}_{\mathrm{tot}}(t)=\sum_{i=1}^{6} \mathbf{r}_{\mathrm{i}}(t) \mathbf{F}_{\mathrm{i}}(t),
$$

where $\mathbf{M}_{\mathrm{tot}}(t)$ represents the total moment about the center of mass, $\mathbf{r}_{\mathrm{i}}(t)$ is a vector from the center of mass to the position of tarsus $i$ and $\mathbf{F}_{\mathrm{i}}(t)$ is the force vector from tarsus $i$.

We calculated the center of pressure of the ground reaction force by using the resultant ground reaction force and moments about the center of mass of the cockroach. We determined the position of a single 'effective' leg by calculating the position at each instant in time, where application of the total ground reaction force vector to the body at the height of the center of mass would produce the same moment about the center of mass as that calculated in $\mathbf{M}_{\text {tot }}$. Thus, the actions of the tripod are replaced by one equivalent leg, which acts with the same resultant force applied at the center of pressure.

From the total moment about the center of mass, the position of the center of pressure was calculated by setting the $z$ (vertical) component of the position equal to the height of the center of mass and by using the total ground reaction force vector (found by summing the components of the three legs over time) to solve the moment equation for the $x, y$ position. The resultant force and moment were replaced by a single force vector applied at a point called the center of pressure.

\section{Results}

\section{Speed and stride frequency}

Data were collected at speeds ranging from 6.3 to $55.5 \mathrm{~cm} \mathrm{~s}^{-1}$ [average speed: $29.9 \pm 14.1 \mathrm{~cm} \mathrm{~s}^{-1}$ (S.D.), $N=23$ ]. Stride frequency increased linearly with speed up to $32 \mathrm{~cm} \mathrm{~s}^{-1}$ and $12.5 \mathrm{~Hz}(P<0.01, N=14)$. Stride frequency did not increase significantly with speed above $32 \mathrm{~cm} \mathrm{~s}^{-1}$ ( $P=0.1, N=9$; Fig. 2A). Stride length remained constant at $2 \mathrm{~cm}$ and did not vary with speed below $32 \mathrm{~cm} \mathrm{~s}^{-1}(P=0.05, N=12$; Fig. 2B), but increased linearly with speed above $32 \mathrm{~cm} \mathrm{~s}^{-1}(P<0.01, N=9$; Fig. 2B).

\section{Duty factor}

In general, duty factors $(\beta)$ were greater than 0.5 , indicating that all six legs contacted the ground for a time during the transition from one tripod of support to another (Fig. 3). The duty factor for the front leg was found to decrease with speed $(P<0.001, N=50$; Fig. 3A). However, at speeds of $32 \mathrm{~cm} \mathrm{~s}^{-1}$ and below, the average duty factor of the front leg was $0.63 \pm 0.01$ (S.E.M.) and did not vary with speed $(P=0.03, N=32)$. At speeds faster 

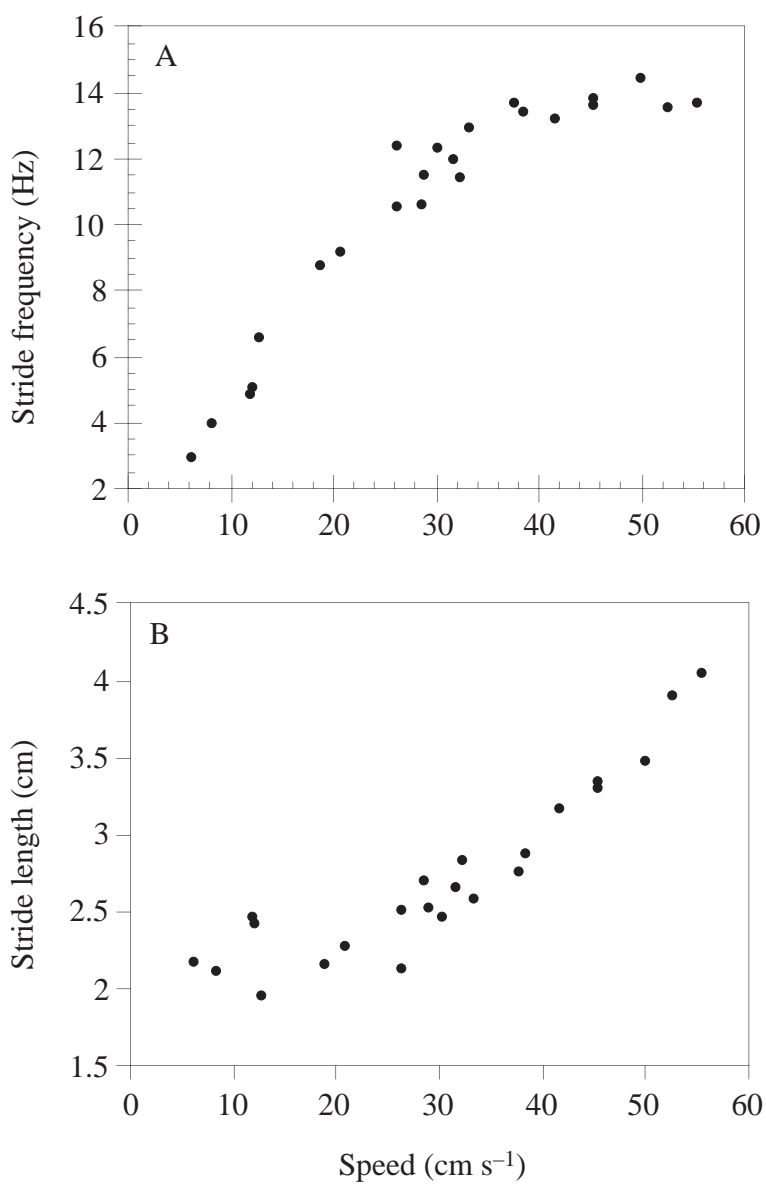

Fig. 2. Stride frequency and stride length as a function of running speed in the cockroach Blaberus discoidalis. (A) Stride frequency, or the rate of leg cycling, increased with speed up to $32 \mathrm{~cm} \mathrm{~s}^{-1}$. At higher speeds, stride frequency did not increase with speed. (B) Stride length, or the forward distance the body moves during a stride, did not change with speed below $32 \mathrm{~cm} \mathrm{~s}^{-1}$. At high speeds, stride length increased with speed.

than $32 \mathrm{~cm} \mathrm{~s}^{-1}$, corresponding to the maximal stride frequency, duty factor decreased with speed $(P<0.001, N=18)$. The duty factor of the middle and hind legs also decreased with speed $(P<0.001, N=50$ for both; Fig. 3B,C), but there was no distinct change in slope at $32 \mathrm{~cm} \mathrm{~s}^{-1}$, as there was with the front leg. The average duty factors for the middle and hind legs $(0.63 \pm 0.01$ and $0.62 \pm 0.01$ respectively) were not significantly different from that for the front leg at speeds slower than $32 \mathrm{~cm} \mathrm{~s}^{-1}$.

\section{Double support and leg phase relationships}

We calculated the amount of double support, when both tripods were on the ground at the same time, as a percentage of the total stride period. The fraction of a stride associated with double support decreased with speed $(P<0.001, N=24$; Fig. 4). During the transition 


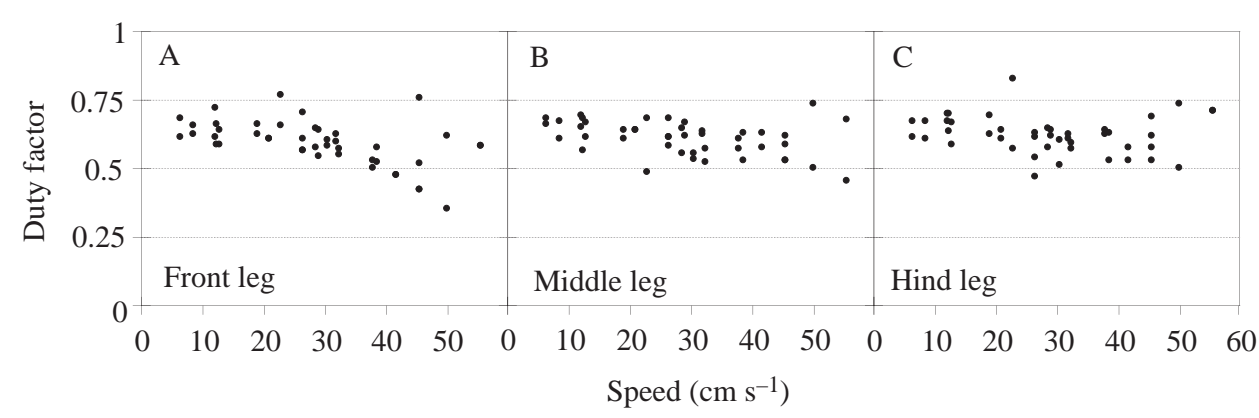

Fig. 3. Duty factor as a function of speed and leg. Duty factor (the fraction of time a leg spends on the ground relative to the stride period) decreased with speed for all legs. (A) The front leg (prothoracic leg) showed little change in duty factor at speeds below $32 \mathrm{~cm} \mathrm{~s}^{-1}$. At higher speeds, duty factor decreased significantly. (B) Middle leg (mesothoracic leg). (C) Hind leg (metathoracic leg).

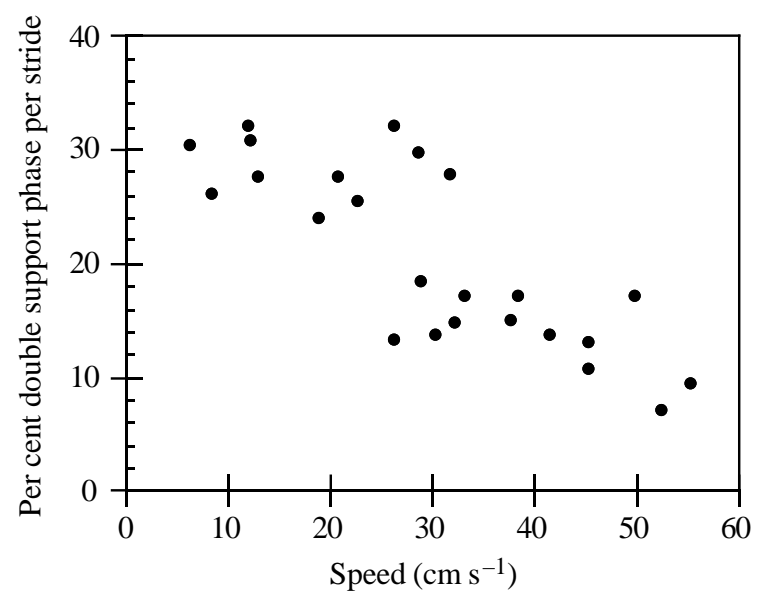

Fig. 4. Per cent double support during a stride as a function of speed. Per cent double support refers to the fraction of a stride that both tripods of support, or all six legs, are in contact with the ground.

from one step to the next at higher speeds, there were periods when fewer than six legs were on the ground during the double support phase.

The cockroaches' gait approximated an alternating tripod at all speeds. The average variation in touch-down timing, or phase, among legs in a tripod was only $4.6 \pm 0.1 \%$ (s.E.M.) of a stride. The touch-down phases (i.e. the time in a stride when a leg first contacts the ground) of the front and middle legs with respect to the hind leg touch-down did not vary with speed $(P>0.4, N=50)$. Lift-off phases (i.e. the time in a stride when a leg lifts off the ground) did decrease with speed, as expected from the duty factor data $(P<0.001$ for all legs; Fig. 5). The decrease in lift-off phase of the front leg with speed was more than twice that in the hind leg.

\section{Stability margin}

The double support period of the stride was instrumental in maintaining high static 


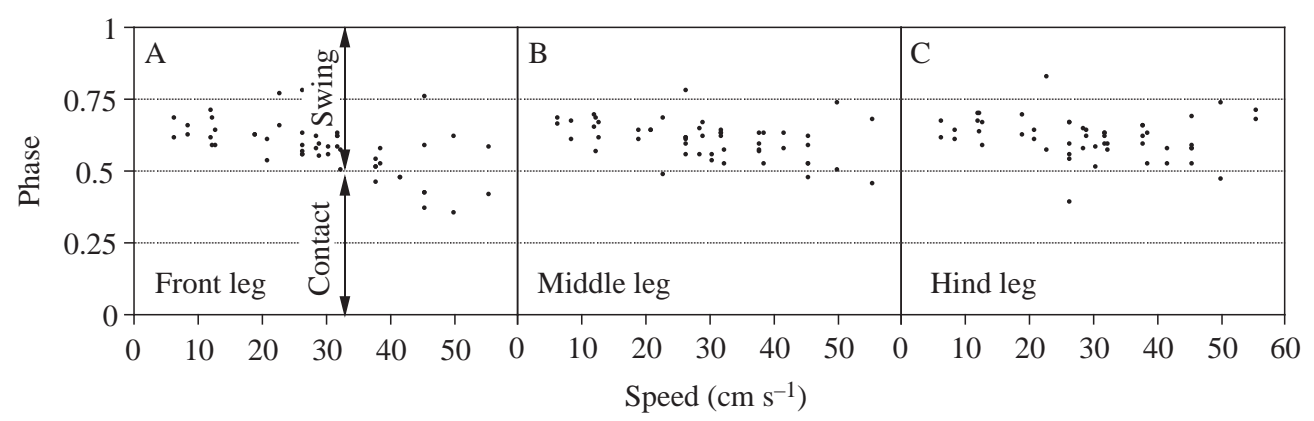

Fig. 5. Phase of leg lift-off as a function of speed and leg. Phase of leg lift-off (the position in a cycle when the leg lifted off the ground, relative to leg touch-down) decreased with speed for all legs. (A) Front leg (prothoracic leg). At the highest speeds, the front leg showed the earliest lift-off phases. (B) Middle leg (mesothoracic leg). (C) Hind leg (metathoracic leg).

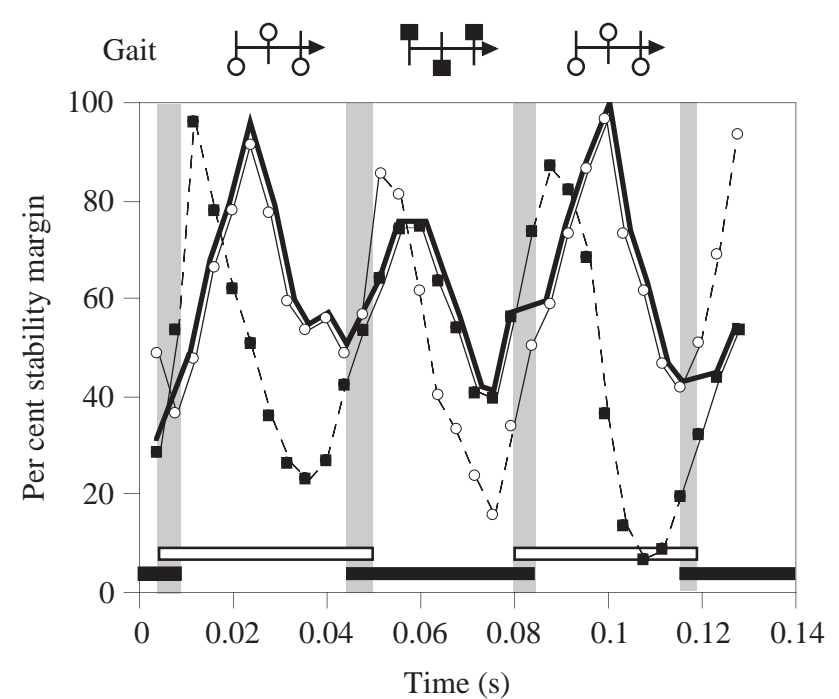

Fig. 6. Per cent stability margin as a function of time. Symbols on the gait diagram represent legs in contact with the ground. Open circles represent one tripod, whereas filled squares represent the alternative tripod. Filled bars show the duration of ground contact by one tripod, whereas open bars show the duration of ground contact of the alternate tripod. Dashed lines connecting the symbols indicate that the tripod is off the ground. This emphasizes the transition of a tripod as it just contacts or leaves the ground. Solid lines connecting the symbols indicate that the tripod is in contact with the ground. The thick solid line represents the per cent stability margin for all legs in contact with the ground. The minimum per cent stability margin is the lowest value of the thick solid line. Shaded vertical bars show periods of double support. In this trial, the cockroach was running at $38.4 \mathrm{~cm} \mathrm{~s}^{-1}$ and showed the lowest per cent stability margin just before the subsequent tripod was placed on the ground.

stability. When all six legs were on the ground, the center of mass could achieve positions farther from the ideal center of stability and still remain within the base of support. The per cent stability margin attained its lowest values near the end of each step, just before the next the tripod landed to provide support (Fig. 6). Low per cent stability margin was most 


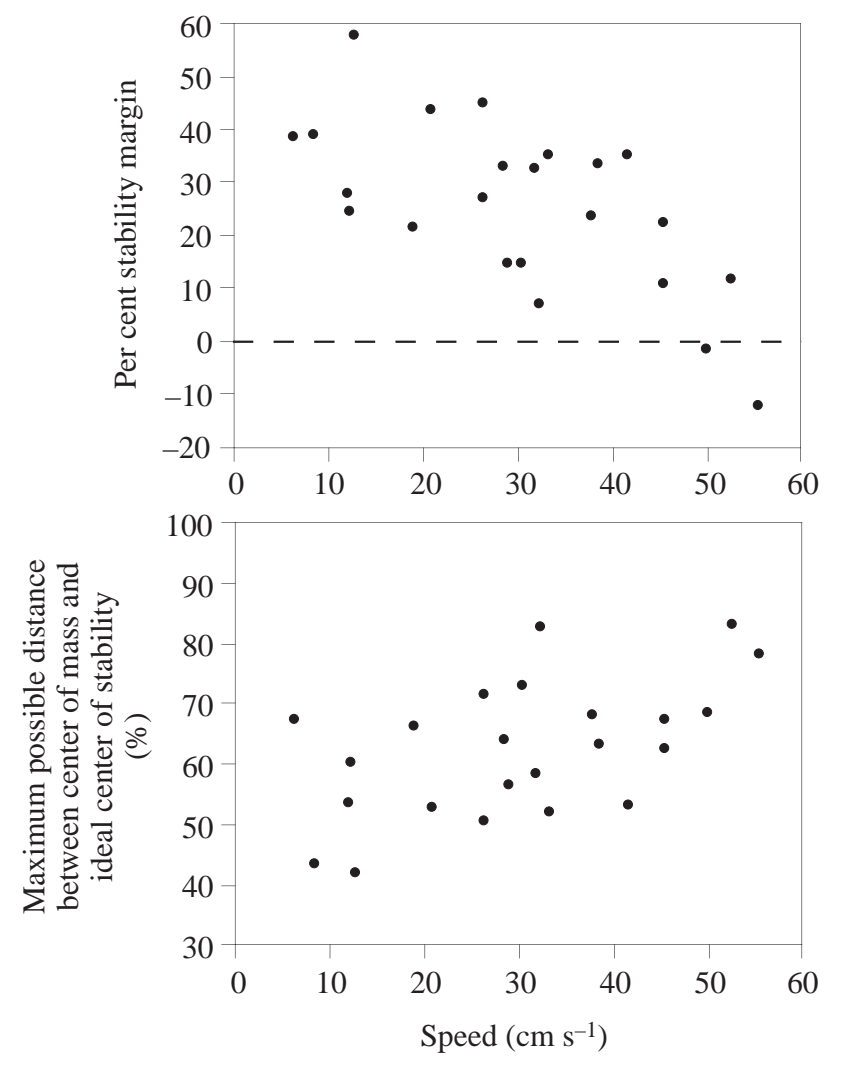

Fig. 7. Per cent stability margin and the longitudinal position of the center of mass as a function of speed. (A) Per cent stability margin decreased with speed. Values less than zero indicate a period of static instability. (B) The maximum possible distance from the center of mass to the ideal center of stability (expressed as a percentage) increased with speed. The center of mass moved rearward or posteriorly as a function of speed.

pronounced at fast speeds, where the double support phase was reduced. During each step, the center of mass started a short distance from the rear boundary of support, moved forwards within the stationary tripod of support and ended each step near the front boundary of support, before entering into the next tripod of support. Thus, the minimum values of the stability margin were measured near the transition from one tripod to the next.

The minimum per cent stability margin attained during a stride decreased with an increase in speed $\left(P=0.005, N=23\right.$; Fig. 7A) and showed no discontinuity at $32 \mathrm{~cm} \mathrm{~s}^{-1}$. The center of mass moved farther away from the ideal center of stability during a step at faster speeds and attained values below zero, indicating static instability.

The longitudinal distance between the center of mass and the ideal center of stability normalized by the maximum possible longitudinal distance increased with speed $(P=0.002, N=23$; Fig. 7B). This resulted from the center of mass being located more posteriorly within the tripod of support as speed increased. The center of mass moved closer to the rear boundary of support and farther from the front boundary as speed increased. 


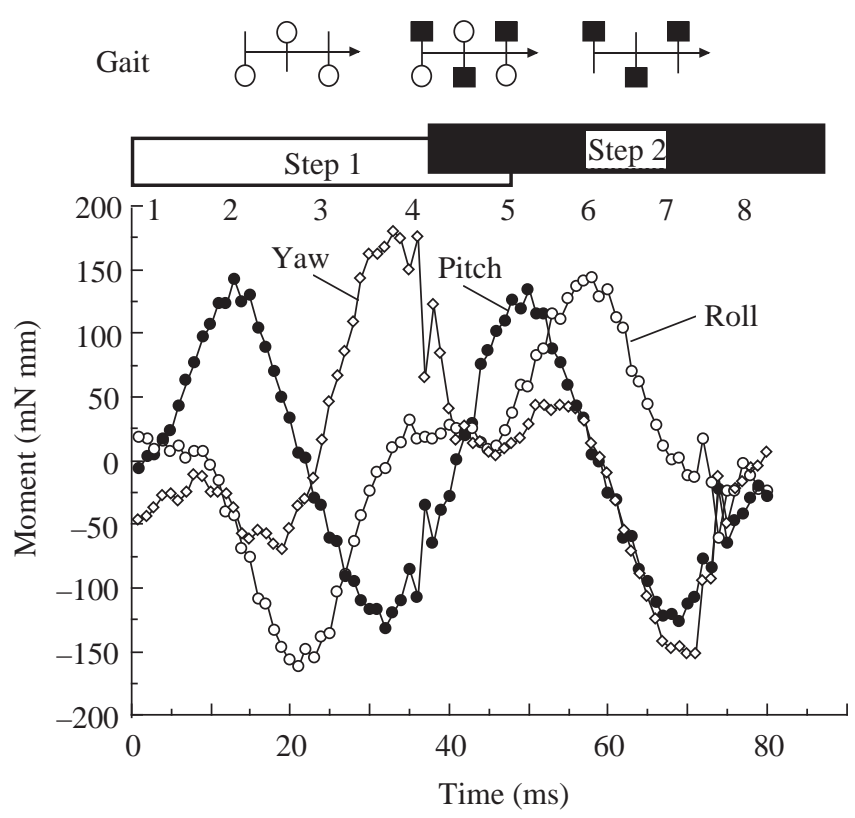

Fig. 8. Moments about the center of mass as a function of time. Symbols on the gait diagram represent legs in contact with the ground. The moments producing body rotation are shown for a cockroach running at $28 \mathrm{~cm} \mathrm{~s}^{-1}$. The numbers shown correspond to periods in the stride shown in Fig. 9. Open diamonds represent the yaw $(z)$ axis, filled circles the pitch axis $(x)$ and open circles the roll axis $(y)$.

\section{Moment of inertia}

The average moments of inertia of the cockroaches were $2.1 \times 10^{-7} \pm 0.2 \times 10^{-7} \mathrm{~kg} \mathrm{~m}^{2}$ (S.D.) about the yaw ( $z$ ) axis, $3.1 \times 10^{-8} \pm 0.4 \times 10^{-8} \mathrm{~kg} \mathrm{~m}^{2}$ (S.D.) about the roll (y) axis and $2.3 \times 10^{-7} \pm 0.5 \times 10^{-7} \mathrm{~kg} \mathrm{~m}^{2}$ (S.D.) about the pitch $(x)$ axis. These values differ little from those of a rectangular prism of similar size to the cockroach that has the density of water.

\section{Moments about the center of mass}

Moments about the center of mass calculated from averaged forces and applied to the kinematic data sets were rather small relative to the absolute variation in moments. Maximum moments about the center of mass generated by the average ground reaction forces were approximately $60-80 \mathrm{mN} \mathrm{mm}$. However, for individual data sets of ground reaction force, the moments generated were quite large, approaching $500 \mathrm{mN} \mathrm{mm}$ about the pitch or $x$-axis (Fig. 8). As the cockroach began each step, inertial moments due to the angular rotation of the animal's body tended to pitch the head downwards (clockwise rotation when running from left to right). During the first half of a step, the inertial moments of the body were opposed by the moments about the $x$-axis due to decelerating ground reaction forces acting in front of the center of mass. The moments due to these ground reaction forces slowed the downward pitching motion of the head and reversed it to an upward pitching motion (counter-clockwise when running from left to right) by 
mid-step. In the second half of the step, the counter-clockwise inertial moments of the body were opposed by the moments about the $x$-axis due to accelerating ground reaction forces acting behind the center of mass. The moments due to these ground reaction forces slowed the upward pitching motion of the head and reversed it to a downward pitching motion by the end of the step. Over each step, the clockwise and counter-clockwise pitching moments balanced one another.

In an analogous situation, the initial inertial moments about the $y$-axis caused the body to roll towards the side with two legs on the ground. As the moment due to the ground reaction force increased, it slowed the rotation towards the side with two legs contacting the ground and reversed the motion by mid-step, so that the animal finished the step rolling towards the side with only one leg down. When the next tripod was set down, the animal once again began the step rolling towards the side with two legs down, and the cycle repeated. During rolling, large moments balanced each other over two steps, or one stride. If the animal began a stride by rolling to the right, then at the end of the first step it was rolling towards the left, and at the end of the second step it was rolling towards the right again.

\section{Center of pressure}

The center of pressure of the ground reaction forces traced a loop about the center of mass over two steps in each trial (Fig. 9). Since the moments generated by averaged ground reaction forces were small, the center of pressure remained very close to the center of mass (Fig. 9A). Over a complete stride, the center of pressure circled symmetrically around the center of mass. For an individual kinematic data set, however, the center of pressure was frequently far from the center of mass (Fig. 9B).

The trajectories observed varied in size and shape, but all showed a very similar pattern. At the beginning of a step, the center of pressure began anterior to the center of mass when the ground reaction forces were small (Fig. 9B, 1-2). The moments were large because the legs were maximally protracted (i.e. extended forward). As the stance phase progressed, the center of pressure moved posterior to the center of mass (Fig. 9B, $2-4)$. The magnitude of the overall moment, which is proportional to the distance from the center of pressure to the center of mass, did not increase despite the increase in force because the legs moved closer to the center of mass. The center of pressure moved rapidly forwards again during the double support phase (Fig. 9B, 4-6) and then backwards again as the next tripod proceeded through stance (Fig. 9B, 6-8). For each step, the position of the center of pressure with respect to the center of mass was balanced in the anterior-posterior direction (i.e. the pitch of the head downwards was balanced by the subsequent pitch of the head upwards). The center of pressure was also balanced in its location lateral to the center of mass, but over two steps, or one stride. The center of pressure was always lateral to the center of mass during stance on the side of the body that had two legs in contact with the ground. During the transition from one tripod to the next, the center of pressure crossed sides so that it remained on the side opposite the middle leg in contact with the ground. Thus, the destabilizing effects of the moments about the center of mass were balanced over an entire stride. 

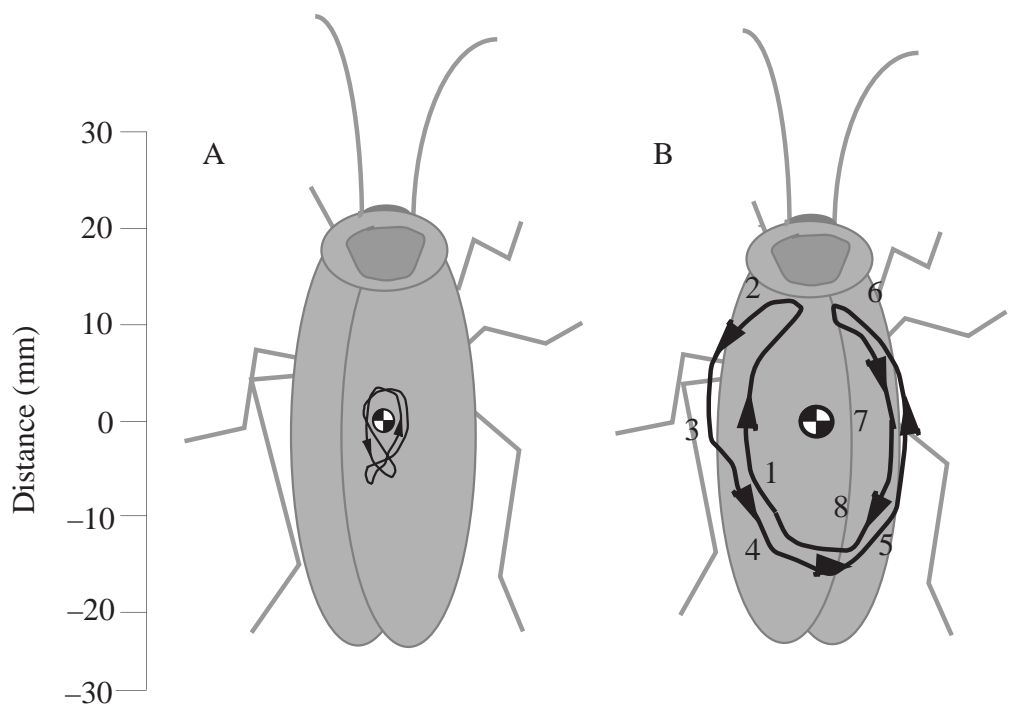

Fig. 9. The path of the center of pressure for several strides and a single stride. The position of the center of pressure (the equivalent of a single 'effective' leg) was calculated from the total moment about the center of mass by setting the $z$ (vertical) component of the position equal to the height of the center of mass and using the total ground reaction force vector (found by summing the components of the three legs over time) to solve the moment equation for $x, y$ position. The resultant force and moment were replaced by a single force vector applied at a point termed the center of pressure. At the beginning of each step, the center of pressure started at a position anterior and lateral to the center of mass on the side opposite the second leg of the tripod. The center of pressure moved posteriorly during stance, ending each step as far posterior of the center of mass as it had been anterior to the center of mass at the beginning of the step, but remaining on the same side lateral to the center of mass. During the transition phase, the center of pressure returned quickly to a position anterior and lateral to the center of mass, but on the opposite side from the previous step. (A) Averaged strides. Trial for cockroach running at $43 \mathrm{~cm} \mathrm{~s}^{-1}$. The center of pressure was close to the center of mass, as expected in static stability. (B) Individual stride. Trial for cockroach running at $45 \mathrm{~cm} \mathrm{~s}^{-1}$. The numbers shown correspond to periods in the stride shown in Fig. 8. The center of pressure was variable and was often far from the center of mass. Over the complete stride, the cockroach was dynamically stable (a push in one direction was compensated for by a push in another).

\section{Discussion}

Legs placed in a sprawled position around the body can provide a more stable posture for resistance against disturbances such as wind (Alexander, 1971). Likewise, an alternating tripod of support with a wide base offers a substantial stability advantage for insects during locomotion. However, using a quasi-static model to characterize insect gaits at all except the slowest speeds is insufficient and can limit the discovery of potential performance advantages of hexapedal designs that capitalize on dynamic behavior.

Speed, stride frequency and whole-body dynamics

The importance of static stability in arthropods can be exaggerated when they are 
compared with dynamically moving mammals at the same stride frequency (Alexander, 1982). Insects are small, operate their legs at higher cycling frequencies and have shorter periods between footfalls than do large mammals. Therefore, the fraction of a stride period required to fall to the ground is not necessarily lower in insects. The American cockroach Periplaneta americana actually requires a greater fraction of a stride to fall (1.1, slightly over a stride period) than does a trotting dog (0.96) when they are compared at stride frequencies normalized to maximum values. The American cockroach cycles its legs at $24 \mathrm{~Hz}$ (i.e. equal to its wing-beat frequency; Full and Tu, 1991), whereas dogs have stride frequencies of about $3 \mathrm{~Hz}$ (Heglund et al. 1974). At these high stride frequencies, Periplaneta americana can run on two or four legs (Full and Tu, 1991). Bipedal running contradicts the hypothesis that static stability is essential for locomotion in insects, since a tripod of support is not used and aerial phases are present. Rapidly sideways-running ghost crabs also show flight phases as they operate two legs on the trailing side of their body (Burrows and Hoyle, 1973; Blickhan and Full, 1987). Maximum stride frequencies used by galloping ghost crabs are similar to rates measured in galloping mice of the same body mass (Blickhan et al. 1993).

Ghost crabs and American cockroaches are exceptionally rapid runners, but the dynamics of their body or center of mass differ little from that of the cockroach in the present study and from those of two- and four-legged mammals (Cavagna et al. 1977; Full and Tu, 1990; Heglund et al. 1982). All can run with a bouncing gait defined by similar ground reaction force patterns (Full, 1989). In B. discoidalis at all speeds and in slower-trotting American cockroaches and ghost crabs, aerial phases are absent, but the dynamics are unmistakably similar to a running gait, as potential and kinetic energy fluctuate in phase. For $B$. discoidalis, the scaling function derived from dynamic gaits of quadrupedal mammals (Heglund et al. 1974; Heglund and Taylor, 1988) predicts both maximum stride frequency and the speed at which it will be attained if the function is extrapolated to small masses (Full and Tu, 1990, 1991). Stepping pattern data alone could never have revealed this dynamic bouncing behavior. Gaits such as walking and running are best defined by the dynamics of the whole body and not simply by an incomplete kinematic data set (Full, 1993).

High-speed running in $B$. discoidalis resulted in stride frequencies of only $13 \mathrm{~Hz}$ (Fig. 2). At these frequencies, the fraction of a stride required to fall to the ground is more similar to that of a slowly walking $\operatorname{dog}(0.4$ at $1.2 \mathrm{~Hz}$; Alexander, 1982). At frequencies below $13 \mathrm{~Hz}$ (i.e. speeds below $30 \mathrm{~cm} \mathrm{~s}^{-1}$ ), falling time is substantially reduced below half a stride period. Although these relative falling times are short compared with those of the fastest arthropods, the dynamics of cockroach locomotion reduces the significance of falling time even at these lower frequencies, because for half of the tripod stance phase the cockroach is being propelled upwards against gravity as it uses a bouncing gait (Full and $\mathrm{Tu}, 1990$ ).

\section{Duty factor and phase}

To maintain static stability in an alternating tripod gait, the duty factor of the legs $(\beta)$ must be greater than or equal to 0.5 [i.e. longitudinal stability, $S=(n / 4) \beta-3 / 4$, is greater than zero if $\beta \geqslant 0.5$ and $N=6$; McGhee, 1976; Song, 1984; Sun, 1974]. Insects seldom use 
duty factors less than 0.5 (Delcomyn, 1971), even though their faster gaits can be considered to be runs, not walks (Full, 1989; Full and Tu, 1990). We measured a mean duty factor of $0.59 \pm 0.07$ (S.D.) (Fig. 3), which represents an average double support of $20.5 \pm 8.0 \%$ (S.D.) for a stride (Fig. 4). The mean duty factor summed over all speeds meets the criterion for static stability. Still, several trends point to the importance of dynamic stability. Duty factors decreased with an increase in speed (Fig. 3). Therefore, the duration of the double support phase also decreased significantly with an increase in speed (Fig. 4). The duty factor in individual legs varied. The front leg had the greatest decline to values equal to or less than 0.5 at high speed. All legs had similar touch-down phases in the tripod. As is typical of insects, the relative phases were generally about 0 , 0.5 and 0 for one side and 0.5, 0 and 0.5 for the other (Delcomyn, 1971). However, lift-off phase was significantly less for the front leg than for the other two legs and can explain part of the change in duty factor (Fig. 5). The decreased time spent in contact with the ground and the early lifting of the front leg relative to the others may result from the fact that the front leg is relatively short. It is only about $15 \mathrm{~mm}$ long, so it is impossible for it to be on the ground for the entire step at higher speeds, as stride length can be as great as $40 \mathrm{~mm}$. We noticed periods during which only the middle and hind legs of the tripod were on the ground, because the front leg had already been lifted. The present data are consistent with a lesser reliance on the front leg and a greater dependence on the middle and hind legs, as in the American cockroach. Periplaneta americana run on their middle and hind legs or only their hind legs with a 'nose up' attitude at their highest speeds (Full and Tu, 1991).

\section{Per cent stability margin}

With the decrease in double support phase, the transition between two steps of the tripod became the least statically stable part of the stride (Fig. 6). If a small degree of double support were not present, then the per cent stability margin would have dropped substantially in all of the trials at the instant before the next tripod was set down. A similar pattern may even be present in slow-moving stick insects (Jander, 1985). As a change occurs from one tripod to the next, the body may be supported by only a hind leg.

As the cockroach increased speed, the minimum per cent stability margin decreased from $60 \%$ of the distance from the edge of support to the most stable position possible at slow speeds to less than zero at high speeds (Fig. 7A). Periods of static instability at the transition from one tripod to the next were evident at high speed. These trends correspond with an increasing significance of dynamics as speed increases. Though slow locomotion could be considered quasi-static, as the dynamic effects at slow speeds were not destabilizing because of the large tripod of support, it appears that adjustments may have been made by the cockroach as speed increased to reduce the destabilizing effects of the increased momentum. The center of mass moved posteriorly within the triangle of support as speed increased (Fig. 7B). The dynamics may be interpreted as a shift which makes the animal less likely to fall by being carried forwards and out of the base of support by inertial forces, should it stop abruptly. The rearward shift of position in the center of mass was also commensurate with the greater reliance on the middle and hind legs at fast speeds suggested by the duty factor and phase data. Such adjustments in the 
position of the center of mass within the tripod of support would not be expected if locomotion could be characterized completely by static measures.

\section{Moments about the center of mass and the center of pressure}

Moments about the center of mass of the cockroach provide further evidence for a significant dynamic component in maintaining stability. Moments were highly variable in phase and magnitude (Fig. 8). Maximum moments (approximately $500 \mathrm{mN} \mathrm{mm}$ ) in the roll axis were equivalent to a force equal to body weight acting on the center of mass at a distance nearly equal to half the body length. If a rolling moment with peak amplitude of $500 \mathrm{mN} \mathrm{mm}$ is applied to a model of the cockroach with a fixed center of mass, the cockroach would spin by more than $360^{\circ}$ in the time it takes to complete one step. The

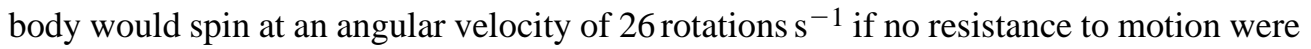
present. Because the body is not simply free to rotate, a more appropriate model for comparison would be a stool with the same dimensions as the tripod formed by the cockroach's three legs.

To this end, we constructed a simple, two-dimensional model of a 'stool' the size of a cockroach and introduced sinusoidal rolling moments with the same amplitude as the rolling moments experienced by the animal. To determine the rotation of the body about the middle leg of the tripod, we used a dynamic simulation program (MATLAB/SIMLAB) to solve the following equation of motion:

$$
\left(I_{\mathrm{zz}}+m l^{2}\right) \ddot{\theta}=M(t)+m \boldsymbol{g} l \cos \theta,
$$

where $I_{\mathrm{zz}}$ is the inertia about the longitudinal axis, $l$ is the distance from the middle leg tarsus to the center of mass, $\theta$ is the angle of rotation of the body from horizontal, $m$ is the mass and $M(t)$ is the moment modeled as half a sinusoid over one step. If the gait of the cockroach were quasi-static, one would expect the moments generated about the middle leg ground contact point not to be large enough to tip the stool against the opposing moment generated by gravity. In the static case, the weight of the animal due to gravity would generate large moments about each ground contact point, which would tend to keep all of the legs on the ground, thus maintaining the center of mass within the tripod of support when subjected to outside disturbances. The moments generated at slow speed, and in the averaged trials, were small and could not tip the 'stool' model of the cockroach. However, if we applied the maximum moments found in the present study (i.e. average peak moment plus two S.D.s), the stool rolled by up to $31^{\circ}$ in one step. The legs of the tripod could not easily remain on the ground with that degree of roll. The peak moment caused by the ground reaction forces was about three times that due to gravity. Thus, these moments would clearly be destabilizing to the cockroach during fast locomotion, if there were no compensation for dynamic effects. Because the cockroach was able to run stably at high speeds, it must be able to balance the dynamic effects of locomotion rather than simply try to prevent the disturbances by providing high static stability. Moments caused the body to roll towards the middle leg of each tripod at the end of each stride, so that the animal was constantly rolling from side to side. Moments in each step decelerated the body in one direction and re-accelerated it in the opposite direction, maintaining dynamic stability over each stride. 
Calculation of the center of pressure showed the dynamic nature of the moments generated about the center of mass. The center of pressure provides the position of a single effective leg in order to visualize better the effect of each tripod (Fig. 9). When the ground reaction forces were averaged, the effective leg operated very near the center of mass, as expected in static situations. When the kinematics of a given stride were examined, then variation in the center of pressure was considerable. Forces acting away from the center of mass generated angular motion in one direction, which was compensated for in the subsequent step. Cockroaches began each step with an initial angular velocity which was reversed during that step or the next.

The data from the present study, taken together with previous studies of insect mechanics (Full and Tu, 1990, 1991; Full et al. 1991), support the contention that dynamic stability is important in hexapedal locomotion, as is static stability. Because cockroaches 'bounce', their position is lowest at midstance and highest at the transition from one tripod to the next. Not only was the cockroach more stable at midstance, as a result of the lower position of the center of mass, but the effective weight (i.e. the vertical force) of the animal was greater as a result of the downward acceleration of the center of mass. Thus, the moments generated by the center of mass about the ground contact points of the limbs were increased, providing greater stability against moments that tended to flip the animal. This period of high stability in the locomotor cycle occurred at the same time as the largest moments generated by the ground reaction forces. During the transition from one tripod to the next, the moments generated by ground reaction forces were lower and the center of mass also had maximal potential and kinetic energy. Although the effective weight of the animal was lower than that due to gravity alone, and the center of mass was in a less stable position, the momentum due to the increased velocity gave the animal high dynamic stability against disturbances and carried it through to the next step. The static stability at this transition point in the stride was lowest and the dynamic stability was the highest. Thus, dynamic stability complemented the static stability provided by the alternating tripod support pattern.

In general, dynamic locomotor systems offer significant advantages over quasi-static systems (Raibert and Hodgins, 1993). A dynamic system can move more rapidly and with less effort over difficult terrain by using balance where only narrow footholds are present, by using kinetic energy to travel where footholds are erratic and by using statically unstable gaits to move over areas where there are no footholds. The kinetic energy of translation and rotation cannot be ignored in the determination of stability in running insects. Kinetic energy can act as a bridge from one step to the next (Raibert and Hodgins, 1993). Rules that govern leg placement can be relaxed in complex environments if locomotion departs from static equilibrium. We predict that the study of the more complex behaviors performed by hexapedal runners, such as climbing and moving over irregular terrain, will require the incorporation of dynamic stability considerations. We contend that robots can be built with six legs for high static stability at slow speeds without necessarily foregoing the advantages of dynamic behavior at high speeds.

We thank Mike Tu very much for his initial effort on the stability calculations. Supported by NSF Grant DCB 89-04586 and ONR Grant NOOO14-92-J-1250. 


\section{References}

AlEXANDER, R. McN. (1971). Size and Shape. London: Edward Arnold.

AlEXANDER, R. MCN. (1982). Locomotion of Animals. Glasow: Blackie.

BlickHAN, R. AND FULl, R. J. (1987). Locomotion energetics of the ghost crab. II. Mechanics of the centre of mass during walking and running. J. exp. Biol. 130, 155-174.

BLICKHAN, R. AND FULL, R. J. (1993). Similarity in multilegged locomotion: Bouncing like a monopode. J. comp. Physiol. 173, 509-517.

Blickhan, R., Full, R. J. AND Ting, L. H. (1993). Exoskeletal strain: evidence for a trot-gallop transition in rapid running ghost crabs. J. exp Biol. 179, 301-321.

Burrows, M. AND HoYle, G. (1973). The mechanism of rapid running in the ghost crab, Ocypode ceratophthalma. J. exp. Biol. 58, 327-349.

Cavagna, G. A., Heglund, N. C. And Taylor, C. R. (1977). Mechanical work in terrestrial locomotion: Two basic mechanisms for minimizing energy expenditure. Am. J. Physiol. 233, R243-R261.

CRuse, H. (1976). The function of legs in the free walking stick insect, Carausius morosus. J. comp. Physiol. 112, 135-162.

Delcomyn, F. (1971). The locomotion of the cockroach Periplaneta americana. J. exp. Biol. 54, 443-452.

Delcomyn, F. (1984). Walking and running. In Comprehensive Insect Physiology, Biochemistry and Pharmacology, vol. 5 (ed. G. A. Kerkut and L. I. Gilbert), pp. 439-466. New York: Pergamon Press.

Delcomyn, F. (1985). Insect locomotion: past, present and future. In Insect Locomotion (ed. M. Gewecke and G. Wendler), pp. 1-18. Berlin: Paul Parey.

Farley, C. T., GlasheEn, J. And MCMahon, T. A. (1993). Running springs: speed and animal size. J. exp. Biol. 185, 71-86.

Fedak, M. A., Heglund, N. C. And Taylor, C. R. (1982). Energetics and mechanics of terrestrial locomotion. II. Kinetic energy changes of the limbs and body as a function of speed and body size in birds and mammals. J. exp. Biol. 79, 23-40.

ForsythE, T. G. (1983). Locomotion in ground beetles (Coleoptera Carabidae): an interpretation of leg structure in functional terms. J. Zool., Lond. 200, 493-507.

Fourtner, C. R. (1976). Central nervous control of cockroach walking. In Neural Control of Locomotion (ed. M. Herman, S. Grillner, P. S. G. Stein and D. G. Styart), pp. 401-418. New York: Plenum Press.

FULL, R. J. (1989). Mechanics and energetics of terrestrial locomotion: From bipeds to polypeds. In Energy Transformation in Cells and Animals (ed. W. Wieser and E. Gnaiger), pp. 175-182. Stuttgart: Thieme Verlag.

FuLL, R. J. (1993). Integration of individual leg dynamics with whole bdy movement in arthropod locomotion. In Biological Neural Networks in Invertebrate Neuroethology and Robotics (ed. R. Beer, R. E. Ritzmann and T. McKenna), pp. 3-20. San Diego: Academic Press.

Full, R. J., Blickhan, R. AND Ting, L. H. (1991). Leg design in hexpedal runners. J. exp. Biol. 158, 369-390.

Full, R. J. AND Koehl, M. A. R. (1993). Drag and lift in running insects. J. exp. Biol. 176, 89-103.

Full, R. J. AND TU, M. S. (1990). The mechanics of six-legged runners. J. exp. Biol. 148, 129-146.

FuLL, R. J. AND TU, M. S. (1991). Mechanics of rapid running insects: two-, four- and six-legged locomotion. J. exp Biol. 156, 215-231.

Graham, D. (1985). Pattern and control of walking in insects. Adv. Insect Physiol. 18, 31-140.

Heglund, N. C., Cavagna, G. A. And Taylor, C. R. (1982). Energetics and mechanics of terrestrial locomotion. III. Energy changes of the center of mass as a function of speed and body size in birds and mammals. J. exp. Biol. 79, 41-56.

Heglund, N. C. AND TAYLOR, C. R. (1988). Speed, stride frequency and energy cost per stride: how do they change with body size and gait? J. exp. Biol. 138, 301-318.

Heglund, N. C., Taylor, C. R. And McMahon, T. A. (1974). Scaling stride frequency and gait to animal size: mice to horses. Science 186, 1112-1113.

Hughes, G. M. (1952). The co-ordination of insect movements. I. The walking movements of insects. J. exp. Biol. 29, 267-285.

Hughes, G. M. (1957). The co-ordination of insect movements. II. The effect of limb amputation and the cutting of commissures in the cockroach (Blatta oreientalis ). J. exp. Biol. 34, 306-333. 
JANDER, J. P. (1985). Mechanical stability of stick insect when walking straight and around curves. In Insect Locomotion (ed. M. Gewecke and G. Wendler). Berlin: Paul Parey.

Manton, S. M. (1977). The Arthropods. London: Clarendon Press.

McGheE, R. B. (1976). Robot locomotion. In Neural Control of Locomotion (ed. M Herman, S. Grillner, P. S. G. Stein and D. G. Styart), pp. 237-264. New York: Plenum Press.

RAibert, M. H. AND Hodgins, J. K. (1993). Legged robots. In Biological Neural Networks in Invertebrate Neuroethology and Robotics (ed. R. Beer, R. E. Ritzmann and T. McKenna), pp. 319-354. San Diego: Academic Press.

SonG, S. M. (1984). Kinematic optimal design of a six-legged walking machine. PhD dissertation, Ohio State University, Columbus, Ohio.

Sun, S. S. (1974). A theoretical study of gaits for legged locomotion systems. PhD dissertation, Ohio State University, Columbus, Ohio. 